\title{
On the Solutions of the Primitive Equations of Motion in the Atmosphere by the Perturbation Method
}

\author{
By M. Magata \\ Meteorological Research Institute \\ (Manuscript received 21 February 1961)
}

\begin{abstract}
It is first demonstrated that the solutions of the primitive equations of motion in both barotropic and baroclinic atmospheres which do not include noticeable meteorological noise can be derived by the perturbation method on the basis of usual geostrophic equations. Namely, the order of magnitude of each term in the equations of motion is estimated using the Rossby number, and the necessary solutions are expanded in the power series of the Rossby number. When the power series does not converge, a method how to treat is then shown especially for the balance equation and this method gives a new method solving the balance equation. It is next shown that when numerical integration is executed by the difference method, particular attention should be paid for an appearance of the secular term. And it is finally shown that though the total energy is conserved in the closed quasi-geostrophic system but it is not conserved without neglecting the quantities of order of the Rossby number in the higher approximate system.
\end{abstract}

\section{Introduction}

Though the quasi-geostrophic model has been used in the numerical weather prediction, many theories and experiments have recently been developed in order to get more accurate forecasts with the primitive equations. The primitive equations generally include three types of solutions corresponding to the acoustic wave, gravity-inertia wave and geostrophic phenomenon. As Hinkelmann (1951) and Phillips (1960) discussed in a linear example, in the numerical weather prediction some proper initial conditions should be taken into account, in order to eliminate small scale waves such as the gravity-inertia waves. Numerical experiments with the primitive equations were made by Smagorinsky (1958) and Hinkelmann (1959). On the other hand Charney (1948), Obukhov (1949) and Gambo (1960) estimated the order of magnitude of each term in the equations of motion and derived the quasi-geostrophic equations as prognostic equations for large scale phenomena. And Charney (1955) and Bolin (1955) have presented balance equation as a genera- lization of the geostrophic relation. Kibel (1958) and Yudin (1959) tried to generalize the quasi-geostrophic theory and to derive the prognostic equations which contain non-geostrophic effects.

In this paper the quasi-geostrophic theory will be generalized by the perturbation method, in order to get more accurate solutions which do not include acoustic and inertiagravity waves. Obukhov (1949) has derived the quasi-geostrophic equations as a first approximation in estimating the order of magnitude of each term in the equations of motion by using the so-called Rossby number. Obukhov's method will more generally be developed by the perturbation method and the equations of higher approximation will be derived. The solutions of the equations of higher approximation will precisely be examined in a linear example and it will be demonstrated that they do not include the gravity-inertia waves. Generally in the perturbation method, solutions of the equations are expanded in the power series of a small parameter such as the Rossby number. But that power series does not always converge 
and though it may converge, the convergent rate may be too slow. A calculation method in that case will be presented with respect to the solution of the balance equation. Another critical point in the perturbation method is the secular term. In the numerical solution by the difference method, the ordinary method (for instance M. Magata 1960) to eliminate the secular term can not be used. Therefore special consideration will be necessary in the process of numerical integration by the difference method.

It is the main aim of the present paper to demonstrate that meteorologically important solutions of the equations of motion of higher approximation than the quasi-geostrophic equations are possible to get by the perturbation method. No numerical example was given and mathematical problems relating to the numerical integration were discussed to some extent.

\section{Mathematical note with respect to the perturbation method}

In this paragraph some fundamental problems relating to the perturbation method will simply be discussed for the ordinary differential equation. The following ordinary differential equation containing a parameter $\lambda$ relating to a function $z$ of a variable $t$ will be considered :

$$
\frac{d z}{d t}=f(t, z, \lambda)
$$

where $f(t, z, \lambda)$ is an analytic function of $t$, $z$ and $\lambda . \quad z=\varphi(t, \lambda)$ is assumed to be a solution of (1) with an initial condition $(z)_{t=0}=C$. A linear equation for

$$
z_{1}=\frac{\partial z}{\partial \lambda}
$$

can be derived by differentiating (1) with $\lambda$ as follows :

$$
\frac{d z_{1}}{d t}=g(t, \lambda) z_{1}+h(t, \lambda)
$$

where

$$
\begin{aligned}
& g(t, \lambda)=\left[\frac{\partial}{\partial z} f(t, z, \lambda)\right]_{z=\varphi(t, \lambda)} \\
& h(t, \lambda)=\left[\frac{\partial}{\partial \lambda} f(t, z, \lambda)\right]_{z=\varphi(t, \lambda)}
\end{aligned}
$$

$z_{1}=\psi(t, \lambda)$ will be assumed to be a solution of (3) with an initial condition $\left(z_{1}\right)_{t=0}=0$. Then the following relation can be proved:

$$
\frac{\partial}{\partial \lambda} \varphi(t, \lambda)=\phi(t, \lambda)
$$

And $\varphi(t, \lambda)$ and $\phi(t, \lambda)$ are analytic functions of $\lambda$. A linear equation for

$$
z_{2}=\frac{\partial^{2} z}{\partial \lambda^{2}}
$$

can be derived by differentiating (1) with $\lambda$ twice as follows :

$$
\begin{aligned}
\frac{d z_{2}}{d t}= & g(t, \lambda) z_{2}+h_{1}(t, \lambda) \phi^{2}(t, \lambda) \\
& +2 h_{2}(t, \lambda) \phi(t, \lambda)+h_{3}(t, \lambda)
\end{aligned}
$$

where

$$
\begin{aligned}
& h_{1}(t, \lambda)=\left[\frac{\partial^{2}}{\partial z^{2}} f(t, z, \lambda)\right]_{z=\varphi(t, \lambda)} \\
& h_{2}(t, \lambda)=\left[\frac{\partial^{2}}{\partial z \partial \lambda} f(t, z, \lambda)\right]_{z=\varphi(t, \lambda)} \\
& h_{3}(t, \lambda)=\left[\frac{\partial^{2}}{\partial \lambda^{2}} f(t, z, \lambda)\right]_{z=\varphi(t, \lambda)}
\end{aligned}
$$

$z_{2}=\chi(t, \lambda)$ is assumed to be a solution of (6) with an initial condition $\left(z_{2}\right)_{t=0}=0$. Then the following relation can be proved:

$$
\frac{\partial^{2}}{\partial \lambda^{2}} \varphi(t, \lambda)=\chi(t, \lambda)
$$

The same relations as (4) and (7) are derivable for $\frac{\partial^{3}}{\partial \lambda^{3}} \varphi(t, \lambda), \frac{\partial^{4}}{\partial \lambda^{4}} \varphi(t, \lambda)$ and so on. In the case of $\lambda=0$ the following power series may be obtained:

$$
z \equiv \varphi(t, \lambda)=\varphi_{0}(t)+\varphi_{1}(t) \lambda+\frac{1}{2 !} \varphi_{2}(t) \lambda^{2}+\cdots \cdots
$$

where

$$
\begin{aligned}
& \varphi_{0}(t)=[\varphi(t, \lambda)]_{\lambda=0} \\
& \varphi_{1}(t)=[\psi(t, \lambda)]_{\lambda=0} \\
& \varphi_{2}(t)=[\chi(t, \lambda)]_{\lambda=0}
\end{aligned}
$$

and these functions are respectively solutions of (1), (3), (6), .... in the case of $\lambda=0$. Of course, these equations can be derived by expanding in the power series of $\lambda$ the both sides of (1) after substituting (8) into (1). Convergence of the series (8) may be too slow. In that case next consideration will be useful. $\lambda=n \Delta \lambda$ is assumed ( $n$ is a proper integer and $\Delta \lambda$ is sufficiently small). $z(t, \Delta \lambda)$ may be obtained from the following relation:

$$
z(t, \Delta \lambda)=z(t, 0)+\Delta \lambda\left[\frac{\partial}{\partial \lambda} z(t, \lambda)\right] \lambda=0
$$


where $z(t, 0)$ and $\left[\frac{\partial}{\partial \lambda} z(t, \lambda)\right]_{\lambda=0}$ can be calculated from (1) and (3). $z(t, 2 \Delta \lambda)$ may be obtained from the following relations :

$$
\left\{\begin{aligned}
z(t, 2 \Delta \lambda)=z(t, \Delta \lambda) & +\Delta \lambda\left[\frac{\partial}{\partial \lambda} z(t, \lambda)\right]_{\lambda=\Delta \lambda} \\
{\left[\frac{\partial}{\partial \lambda} z(t, \lambda)\right]_{\lambda=\Delta \lambda}=} & {\left[\frac{\partial}{\partial \lambda} z(t, \lambda)\right]_{\lambda=0} } \\
& +\Delta \lambda\left[\frac{\partial^{2}}{\partial \lambda^{2}} z(t, \lambda)\right]_{\lambda=0}
\end{aligned}\right.
$$

In the same way $z(t, 3 \Delta \lambda), \cdots \cdots, z(t, n \Delta \lambda)$ may be obtained. It will later be demonstrated for the balance equation that this method is more useful than the power series such as (8). The above method will be applied to the partial differential equations of motion in the following.

\section{Generalization of the quasi-geostrophic theory in the barotropic case}

The barotropic case will first be considered and our method will precisely be discussed for this case. If $x^{\prime}$ and $y^{\prime}$ are rectangular horizontal coordinates and $u^{\prime}$ and $v^{\prime}$ the corresponding velocity components, the equations of motion for the barotropic case become

$$
\left\{\begin{array}{l}
\frac{\partial u^{\prime}}{\partial t^{\prime}}+u^{\prime} \frac{\partial u^{\prime}}{\partial x^{\prime}}+v^{\prime} \frac{\partial u^{\prime}}{\partial y^{\prime}}-f^{\prime} v^{\prime}=-\gamma^{\prime 2} \frac{\partial \sigma^{\prime}}{\partial x^{\prime}} \\
\frac{\partial v^{\prime}}{\partial t^{\prime}}+u^{\prime} \frac{\partial u^{\prime}}{\partial x^{\prime}}+v^{\prime} \frac{\partial v^{\prime}}{\partial y^{\prime}}+f^{\prime} u^{\prime}=-\gamma^{\prime 2} \frac{\partial \sigma^{\prime}}{\partial y^{\prime}} \\
\frac{\partial \sigma^{\prime}}{\partial t^{\prime}}+u^{\prime} \frac{\partial \sigma^{\prime}}{\partial x^{\prime}}+v^{\prime} \frac{\partial \sigma^{\prime}}{\partial y^{\prime}}+\frac{\partial u^{\prime}}{\partial x^{\prime}}+\frac{\partial v^{\prime}}{\partial y^{\prime}}=0
\end{array}\right.
$$

where $\sigma^{\prime}=\ln p_{G}, p_{G}$ is surface pressure and $\gamma^{\prime 2}=g H, H$ being mean atmospheric height. In general, all dimensional variables will be denoted by primes. Let $L$ be the horizontal characteristic length, $U$ the horizontal characteristic particle velocity and the characteristic phase velocity of the disturbances is assumed to be equal to $U$. Then the nondimensional quantities $x, y, t, u, v, \sigma$ and $f$ may be defined as follows:

$$
\begin{aligned}
& x=\frac{x^{\prime}}{L}, \quad y=\frac{y^{\prime}}{L}, \quad t=\frac{U}{L} t^{\prime}, \quad u=\frac{u^{\prime}}{U}, \\
& v=\frac{v^{\prime}}{U}, \quad f=\frac{f^{\prime}}{2 \Omega}, \quad \sigma=\frac{\gamma^{\prime 2} \sigma^{\prime}}{2 \Omega U L}
\end{aligned}
$$

where $\Omega$ is the angular speed of the earth's rotation. The Rossby number $R=U / 2 \Omega L$ which measures the ratio of the relative horizontal acceleration to the horizontal Coriolis force, is small when the large scale geostrophic disturbances are considered. The equations of motion (11) may be written as follows by using (12):

$$
\left\{\begin{array}{l}
R\left(\frac{\partial u}{\partial t}+u \frac{\partial u}{\partial x}+v \frac{\partial u}{\partial y}\right)-f v=-\frac{\partial \sigma}{\partial x} \\
R\left(\frac{\partial v}{\partial t}+u \frac{\partial v}{\partial x}+v \frac{\partial v}{\partial y}\right)+f u=-\frac{\partial \sigma}{\partial y} \\
R\left(\frac{\partial \sigma}{\partial t}+u \frac{\partial \sigma}{\partial x}+v \frac{\partial \sigma}{\partial y}\right)+\frac{1}{\mu^{2}}\left(\frac{\partial u}{\partial x}+\frac{\partial v}{\partial y}\right)
\end{array}\right.
$$

where

$$
R=\frac{U}{2 \Omega L}, \quad \mu=\frac{2 \Omega L}{\gamma^{\prime 2}}
$$

$u, v$ and $\sigma$ may be considered as unknown functions of variables $t, x, y$ and parameter $R$. $u$ and $v$ may be expressed by using the stream function $\phi$ and velocity potential $\chi$ as follows :

$$
\left\{\begin{array}{l}
u=-\frac{\partial \dot{\psi}}{\partial y}+\frac{\partial \chi}{\partial x} \\
v=-\frac{\partial \dot{\psi}}{\partial x}+\frac{\partial \chi}{\partial y}
\end{array}\right.
$$

The solutions of (13) will be assumed to be expansible in the power series of $R$ as follows according to the previous paragraph:

$$
\left\{\begin{aligned}
& u=u_{0}+R u_{1}+R^{2} u_{2}+\cdots \cdots \\
& v=v_{0}+R v_{1}+R^{2} v_{2}+\cdots \cdots \\
& \phi=\phi_{0}+R \phi_{1}+R^{2} \phi_{2}+\cdots \cdots \\
& \chi=\chi_{0}+R \chi_{1}+R^{2} \chi_{2}+\cdots \cdots \\
& \sigma=\sigma_{0}+R \sigma_{1}+R^{2} \sigma_{2}+\cdots \cdots \\
& u_{n}=-\frac{\partial \dot{\varphi}_{n}}{\partial y}+\frac{\partial \chi_{n}}{\partial x}, \quad v_{n}=\frac{\partial \phi_{n}}{\partial x}+\frac{\partial \chi_{n}}{\partial y} \\
& \quad[n=0,1,2, \cdots \cdots]
\end{aligned}\right.
$$

(16) is substituted into (15), and (15) is substituted into the equation (13). And if the coefficients of the same power of $R$ are set equal, the following systems of equations may be obtained :

$$
\left\{\begin{array}{c}
-f v_{0}=-\frac{\partial \sigma_{0}}{\partial x} \\
f u_{0}=-\frac{\partial \sigma_{0}}{\partial y} \\
\frac{\partial u_{0}}{\partial x}+\frac{\partial v_{0}}{\partial y}=0
\end{array}\right.
$$

$$
\left\{\begin{array}{l}
\frac{\partial u_{0}}{\partial t}+u_{0} \frac{\partial u_{0}}{\partial x}+v_{0} \frac{\partial u_{0}}{\partial y}-f v_{1}=-\frac{\partial \sigma_{1}}{\partial x} \\
\frac{\partial v_{0}}{\partial t}+u_{0} \frac{\partial v_{0}}{\partial x}+v_{0} \frac{\partial v_{0}}{\partial y}+f u_{1}=-\frac{\partial \sigma_{1}}{\partial y}
\end{array}\right.
$$




$$
\left\{\begin{array}{c}
\frac{\partial \sigma_{0}}{\partial t}+u_{0} \frac{\partial \sigma_{0}}{\partial x}+v_{0} \frac{\partial \sigma_{0}}{\partial y}+\frac{1}{\mu^{2}}\left(\frac{\partial u_{1}}{\partial x}+\frac{\partial v_{1}}{\partial y}\right)=0 \\
=-\frac{\partial \sigma_{2}}{\partial x} \\
\frac{\partial u_{1}}{\partial t}+u_{0} \frac{\partial u_{1}}{\partial x}+u_{1} \frac{\partial u_{0}}{\partial x}+v_{0} \frac{\partial u_{1}}{\partial y}+v_{1} \frac{\partial u_{0}}{\partial y}-f v_{2} \\
\frac{\partial v_{1}}{\partial t}+u_{0} \frac{\partial v_{1}}{\partial x}+u_{1} \frac{\partial v_{0}}{\partial x}+v_{0} \frac{\partial v_{1}}{\partial y}+v_{1} \frac{\partial v_{0}}{\partial y}+f u_{2} \\
=-\frac{\partial \sigma_{2}}{\partial y} \\
\frac{\partial \sigma_{1}}{\partial t}+u_{0} \frac{\partial \sigma_{1}}{\partial x}+u_{1} \frac{\partial \sigma_{0}}{\partial x}+v_{0} \frac{\partial \sigma_{1}}{\partial y}+v_{1} \frac{\partial \sigma_{0}}{\partial y} \\
+\frac{1}{\mu^{2}}\left(\frac{\partial u_{2}}{\partial x}+\frac{\partial v_{2}}{\partial y}\right)=0
\end{array}\right.
$$

In the same way the equations of more higher order can be obtained. From (17) and (16)

$$
\left\{\begin{array}{l}
\phi_{0}=\frac{1}{f} \sigma_{0} \\
\chi_{0}=0
\end{array}\right.
$$

Therefore

$$
u_{0} \frac{\partial \sigma_{0}}{\partial x}+v_{0} \frac{\partial \sigma_{0}}{\partial y}=0
$$

And the third equation of (18) may be written as follows :

$$
\frac{\partial \sigma_{0}}{\partial t}+\frac{1}{\mu^{2}} \nabla^{2} \chi_{1}=0
$$

If the vorticity equation is produced from (18), the following quasi-geostrophic equation can be obtained by using (17), (20) and (21):

$$
\nabla^{2} \frac{\partial \psi_{0}}{\partial t}-(f \mu)^{2} \frac{\partial \phi_{0}}{\partial t}=J\left(\nabla^{2} \psi_{0}+f, \phi_{0}\right)
$$

If the divergence equation is produced from (18), the following relation corresponding to the balance equation may be obtained:

$$
2\left(\frac{\partial^{2} \psi_{0}}{\partial x^{2}} \frac{\partial^{2} \psi_{0}}{\partial y^{2}}-\left(\frac{\partial^{2} \psi_{0}}{\partial x \partial y}\right)^{2}\right)+f \nabla^{2} \psi_{1}=\nabla^{2} \sigma_{1}
$$

Thus the first approximation of the solutions of the equations (13) can be obtained from the quasi-geostrophic equation (22). Now the second approximation of the solutions of the equations (13) will be inquired. If the equation corresponding to the vorticity equation is produced from the equations (19),

$$
\begin{gathered}
\frac{\partial}{\partial t} \nabla^{2} \psi_{1}+J\left(\psi_{0}, \nabla^{2} \psi_{1}\right)+J\left(\psi_{1}, \nabla^{2} \psi_{0}\right) \\
+\frac{\partial \chi_{1}}{\partial x} \frac{\partial}{\partial x} \nabla^{2} \psi_{0}+\frac{\partial \chi_{1}}{\partial y} \frac{\partial}{\partial y} \nabla^{2} \psi_{0} \\
+\nabla^{2} \psi_{0} \cdot \nabla^{2} \chi_{1}+f \nabla^{2} \chi_{2}=0
\end{gathered}
$$

The third equation of (19) may be written as follows :

$$
\begin{aligned}
& \frac{\partial \sigma_{1}}{\partial t}+J\left(\phi_{0}, \sigma_{1}\right)+J\left(\phi_{1}, \sigma_{0}\right) \\
& +\frac{\partial \chi_{1}}{\partial x} \frac{\partial \sigma_{0}}{\partial x}+\frac{\partial \chi_{1}}{\partial y} \frac{\partial \sigma_{0}}{\partial y}+\frac{1}{\mu^{2}} \nabla^{2} \chi_{2}=0
\end{aligned}
$$

The following prognostic equation for $\phi_{1}$ can be derived from (23), (24) and (25):

$$
\frac{\partial}{\partial t} \nabla^{2} \psi_{1}-(f \mu)^{2} \frac{\partial \phi_{1}}{\partial t}=F \text {. }
$$

where $F$ can be calculated from the values of $\phi_{0}$ and $\psi_{1}$. As initial conditions, $\left(\sigma_{0}\right)_{t=0}$ or $\left(\psi_{0}\right)_{t=0}$ and $\left(\psi_{1}\right)_{t=0}=\left(\psi_{2}\right)_{t=0}=\cdots \cdots=0$ should be given. Thus the solutions of the equations (13) till the second approximation of the Rossby number $R$ will be obtained. The solutions of higher approximations may in the same way be inquired. But a noteworthy point is that in order to get the solutions of the second approximation, the geostrophic vorticity equation should not first be solved till the necessary time and then solve (26) by using the obtained $\phi_{0}$, because the solution of (26) may become infinite with time by the effect of the so-called secular term. The equations (22) and (26) should be considered as a system of simultaneous equations only in the sufficiently short time interval. Namely let the solution at $t=t_{0}+\Delta t$ be $\phi_{0}=h_{0}(x, y)$ and $\phi_{1}=h_{1}(x, y)$ with the initial conditions $\left(\psi_{0}\right)_{t=t_{0}}=f_{0}(x, y)$ and $\left(\psi_{1}\right)_{t=t_{0}}=0$. Then in the next time stage the calculation should be done with the initial conditions $\left(\psi_{0}\right)_{t=t_{0}+\Delta t}=h_{0}+R h_{1}$ and $\left(\phi_{1}\right)_{t=t_{0}+\Delta t}=0$. The same remark should also be given in the numerical calculation' of the solution of higher approximation.

Now in order to know what is the characteristic feature of the solutions of the type (16), the linearized equations of (13) will in the next paragraph be considered.

\section{Linear system in the barotropic case}

It is difficult to discuss exactly what kind of solutions of the primitive equations (13) are the solutions of type (16), especially whether they include the gravity-inertia waves or not. Therefore in this paragraph the linearized equations of (13) will be discussed and the characteristic features of the solutions of type (16) will be inquired. $U$ and $c-f U y$ are assumed to be uniform general current and the corresponding distribution of 
$\sigma$ respectively. Then the linearized equations of (13) for the deviations of $u, v$ and $\sigma$ from the general current may be written as follows:

$$
\left\{\begin{array}{l}
R\left(\frac{\partial u}{\partial t}+U \frac{\partial u}{\partial x}\right)-f v=-\frac{\partial \sigma}{\partial x} \\
R\left(\frac{\partial v}{\partial t}+U \frac{\partial v}{\partial x}\right)+f u=0 \\
R\left(\frac{\partial \sigma}{\partial t}+U \frac{\partial \sigma}{\partial x}-f U v\right)+\frac{1}{\mu^{2}} \frac{\partial u}{\partial x}=0
\end{array}\right.
$$

where the deviations of $u, v$ and $\sigma$ from the general current are again denoted by $u, v$ and $\sigma$, and these quantities are assumed to be functions of $x$ and $t$ only. The general solutions of the linear system (27) will first be inquired. The following wave-solutions will be considered :

$$
\left\{\begin{array}{l}
u=u^{*} e^{i k(x-c t)} \\
v=v^{*} e^{i k(x-c t)} \\
\sigma=\sigma^{*} e^{i k(x-c t)}
\end{array}\right.
$$

where $k$ is wave-number, $c$ phase-velocity and $u^{*}, v^{*}$ and $\sigma^{*}$ are amplitudes. If (28) is substituted into the equations (27) and they are rearranged, the following relations will be obtained :

$$
\left\{\begin{array}{l}
R(c-U) k u^{*}-i f v^{*}-k \sigma^{*}=0 \\
i f u^{*}+R(c-U) k v^{*}=0 \\
\frac{k}{\mu^{2}} u^{*}+i R f U v^{*}-R(c-U) k \sigma^{*}=0
\end{array}\right.
$$

In order that $u^{*}, v^{*}$ and $\sigma^{*}$ satisfying (29) are not all zero, the following relation is necessary :

$$
\left|\begin{array}{ccc}
R(c-U) k & -i f & -k \\
\text { if } & R(c-U) k & 0 \\
\frac{k}{\mu^{2}} & i R f U & -R(c-U) k
\end{array}\right|=0
$$

which determines the phase-velocity $c$. may be written as follows:

$$
k^{2} X^{3}-\left(f^{2}+\frac{k^{2}}{\mu^{2}}\right) X-R f^{2} U=0
$$

where

$$
X=R(c-U)
$$

The following relations will be obtained from (29) for $X$ or $c$ determined from (31):

$$
\left\{\begin{array}{l}
u^{*}=\frac{k^{2} X}{k^{2} X^{2}-f^{2}} \sigma^{*} \\
v^{*}=-\frac{i k f}{k^{2} X^{2}-f^{2}} \sigma^{*}
\end{array}\right.
$$

There are three roots of the algebraic equation (31) which are denoted by $X_{1}, X_{2}$ and $X_{3}$. When $R=0$, the equation (31) becomes as follows :

$$
X\left\{k^{2} X^{2}-\left(f^{2}+\frac{k^{2}}{\mu^{2}}\right)\right\}=0
$$

three roots of which are $X=0, \pm \sqrt{\frac{f^{2}}{k^{2}}+\frac{1}{\mu^{2}}}$. Thus the following relations will be assumed :

$$
\left\{\begin{array}{l}
\left(X_{1}\right)_{R=0}=0 \\
\left(X_{2}\right)_{R=0}=\sqrt{\frac{f^{2}}{k^{2}}+\frac{1}{\mu^{2}}} \\
\left(X_{3}\right)_{R=0}=-\sqrt{\frac{f^{2}}{k^{2}}+\frac{1}{\mu^{2}}}
\end{array}\right.
$$

where $X_{2}$ and $X_{3}$ correspond to the phasevelocities of the gravity-inertia waves. If (32) is substituted into (31),

$$
R^{2} k^{2}(c-U)^{3}-\left(f^{2}+\frac{k^{2}}{\mu^{2}}\right)(c-U)-f^{2} U=0
$$

If $R=0$,

$$
c-U=-\frac{f^{2} U}{f^{2}+\frac{k^{2}}{\mu^{2}}} \text { or } c=\frac{k^{2} / \mu^{2}}{f^{2}+\frac{k^{2}}{\mu^{2}}} U
$$

which is the phase velocity of the meteorological important wave and corresponds to $X_{1}$ of (34). If $X_{1}$ is used as a value of $X$ in (33),

$$
\begin{aligned}
& \left(u^{*}\right)_{R=0}=0 \\
& \left(v^{*}\right)_{R=0}=\frac{i k}{f} \sigma^{*}
\end{aligned}
$$

which correspond to the geostrophic relation as may be seen from (27) and (28). But if $X_{2}$ or $X_{3}$ is used in (33), the geostrophic relation cannot be obtained when $R=0$. The solutions of type (16) in the previous paragraph are the solutions which become geostrophic when $R=0$. Therefore in the linear system (27) the meteorologically important solution can be expressed in the form as (16), but the gravity-inertia waves cannot be expressed in such form as (16). It may thus be inferred also in the nonlinear equations (13) that the solutions of type (16) express the meteorologically important solutions which do not include the gravity-inertia waves.

\section{Generalization of the quasi-geostrophic theory in the baroclinic case}

The perturbation method will now be applied to the baroclinic case, and the first ap- 
proximation will be demonstrated to be the quasi-geostrophic equations. According to Charney's method (1960), the dimensional analysis is first made by assigning characteristic values to the physical magnitudes appearing in the equations of motion and with their aid the equations are written in nondimensional form. The equations of motion, continuity and thermodynamics in the baroclinic atmosphere may be written as follows:

$$
\left\{\begin{array}{l}
\frac{\partial u^{\prime}}{\partial t^{\prime}}+u^{\prime} \frac{\partial u^{\prime}}{\partial x^{\prime}}+v^{\prime} \frac{\partial u^{\prime}}{\partial y^{\prime}}+\omega^{\prime} \frac{\partial u^{\prime}}{\partial p^{\prime}}-f^{\prime} v^{\prime}=-\frac{\partial \phi^{\prime}}{\partial x^{\prime}} \\
\frac{\partial v^{\prime}}{\partial t^{\prime}}+u^{\prime} \frac{\partial v^{\prime}}{\partial x^{\prime}}+v^{\prime} \frac{\partial v^{\prime}}{\partial y^{\prime}}+\omega^{\prime} \frac{\partial v^{\prime}}{\partial p^{\prime}}+f^{\prime} u^{\prime}=-\frac{\partial \phi^{\prime}}{\partial y^{\prime}} \\
\frac{\partial u^{\prime}}{\partial x^{\prime}}+\frac{\partial v^{\prime}}{\partial y^{\prime}}+\frac{\partial \omega^{\prime}}{\partial p^{\prime}}=0 \\
\frac{\partial}{\partial t^{\prime}}\left(\frac{\partial \phi^{\prime}}{\partial p^{\prime}}\right)+u^{\prime} \frac{\partial}{\partial x^{\prime}}\left(\frac{\partial \phi^{\prime}}{\partial p^{\prime}}\right)+v^{\prime} \frac{\partial}{\partial y^{\prime}}\left(\frac{\partial \phi^{\prime}}{\partial p^{\prime}}\right)+s^{\prime} \omega^{\prime}=0
\end{array}\right.
$$

where $\omega^{\prime}$ is the individual derivative of pressure, $\phi^{\prime}$ the geopotential, $p^{\prime}$ the pressure and $s^{\prime}$ the coefficient of static stability. All dimensional variables of the equations will again be denoted by primes. Let $L$ be the horizontal characteristic length, $U$ the horizontal characteristic particle velocity and the characteristic phase velocity of the disturbances is assumed to be equal to $U$. In that case the characteristic time is equal to $L / U$. Further let $D$ be the characteristic pressure and $\kappa$ the characteristic static stability. Then the nondimensional quantities $x, y, p, t, u, v, s$ and $f$ may be defined as follows:

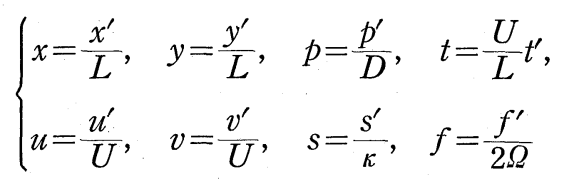

where $\Omega$ is the angular speed of the earth's rotation, and $u, v, s$ and $f$ have the order of magnitude unity. Next the characteristic quantities of $\phi^{\prime}$ and $\omega^{\prime}$ will be estimated. From the geostrophic balance it may be seen that $2 \Omega L U$ is a measure of the characteristic variation of $\phi^{\prime}$ in an isobaric surface $i . e$., if $\phi_{s}$ denotes the standard isobaric average of $\phi^{\prime}$,

$$
\phi=\frac{\phi^{\prime}-\phi_{s}}{2 \Omega L U}
$$

defines a non-dimensional geopotential with the order of magnitude unity. The characteristic individual change of pressure may be estimated from the equation of thermodynamics by using (36) and (37). Thus

$$
\omega=\frac{\kappa D}{2 \Omega U^{2}} \omega^{\prime}
$$

defines a non-dimensional quantity of $\omega^{\prime}$ with the order of magnitude unity. If (36), (37) and (38) are used, the equations of motion, continuity and thermodynamics may be written as follows:

$$
\left\{\begin{array}{l}
R\left(\frac{\partial u}{\partial t}+u \frac{\partial u}{\partial x}+v \frac{\partial u}{\partial y}\right)+\lambda R^{2} \omega \frac{\partial u}{\partial p}-f v=-\frac{\partial \phi}{\partial x} \\
R\left(\frac{\partial v}{\partial t}+u \frac{\partial v}{\partial x}+v \frac{\partial v}{\partial y}\right)+\lambda R^{2} \omega \frac{\partial v}{\partial p}+f u=-\frac{\partial \phi}{\partial y} \\
\frac{\partial u}{\partial x}+\frac{\partial v}{\partial y}+\lambda R \frac{\partial \omega}{\partial p}=0 \\
R\left(\frac{\partial}{\partial t}\left(\frac{\partial \phi}{\partial p}\right)+u \frac{\partial}{\partial x}\left(\frac{\partial \phi}{\partial p}\right)+v \frac{\partial}{\partial y}\left(\frac{\partial \phi}{\partial p}\right)+s \omega\right)=0
\end{array}\right.
$$

where

$$
\left\{\begin{array}{l}
R=\frac{U}{2 \Omega L} \\
\lambda=\frac{4 \Omega^{2} L^{2}}{\kappa D^{2}}
\end{array}\right.
$$

The solutions of (39) may be expanded in the power series of $R$ as follows :

$$
\left\{\begin{array}{c}
u=u_{0}+R u_{1}+R^{2} u_{2}+\cdots \cdots \\
v=v_{0}+R v_{1}+R^{2} v_{2}+\cdots \cdots \\
\omega=\omega_{0}+R \omega_{1}+R^{2} \omega_{2}+\cdots \cdots \\
\phi=\phi_{0}+R \phi_{1}+R^{2} \phi_{2}+\cdots \cdots \\
\phi=\phi_{0}+R \phi_{1}+R^{2} \phi_{2}+\cdots \cdots \\
\chi=\chi_{0}+R \chi_{1}+R^{2} \chi_{2}+\cdots \cdots
\end{array}\right.
$$

where $\phi$ and $\chi$ are the stream function and the velocity potential respectively, which are connected with $u$ and $v$ by the relations (15). If (41) is substituted into the equations (39) in the same way with the barotropic case, the quasi-geostrophic equations can as the first approximation be derived as follows:

$$
\left\{\begin{array}{l}
\chi_{0}=0, \quad \phi_{0}=\frac{1}{f} \phi_{0} \\
\frac{\partial}{\partial t} \nabla^{2} \phi_{0}-J\left(\nabla^{2} \phi_{0}, \phi_{0}\right)-\lambda f^{2} \frac{\partial \omega_{0}}{\partial p}=0 \\
\frac{\partial}{\partial t}\left(\frac{\partial \phi_{0}}{\partial p}\right)-J\left(\frac{\partial \phi_{0}}{\partial p}, \phi_{0}\right)+s \omega_{0}=0 \\
\nabla^{2} \chi_{1}+\lambda \frac{\partial \omega_{0}}{\partial p}=0
\end{array}\right.
$$

And the first approximation of the divergence equation is 


$$
2\left(\frac{\partial^{2} \dot{\psi}_{0}}{\partial x^{2}} \frac{\partial^{2} \psi_{0}}{\partial y^{2}}-\left(\frac{\partial^{2} \psi_{0}}{\partial x \partial y}\right)^{2}\right)+f \nabla^{2} \psi_{\mathrm{L}}=\nabla^{2} \phi_{1}
$$

In (42) and (43), latitude change of $f$ was ignored. The so-called $\omega$-equation may be written as follows:

$$
\begin{aligned}
& s V^{2} \omega_{0}+\lambda f^{2} \frac{\partial^{2} \omega_{0}}{\partial p^{2}} \\
& \quad=\nabla^{2}\left\{J\left(\frac{\partial \phi_{0}}{\partial p}, \phi_{0}\right)\right\}-\frac{\partial}{\partial p} J\left(\nabla^{2} \dot{\phi}_{0}, \phi_{0}\right)
\end{aligned}
$$

where $s$ was assumed constant.

The second approximation of the equations of vorticity, thermodynamics and continuity can be written as follows :

$$
\left\{\begin{array}{l}
\frac{\partial}{\partial t} \nabla^{2} \phi_{1}-J\left(\nabla^{2} \phi_{1}, \phi_{0}\right)-J\left(\nabla^{2} \phi_{0}, \phi_{1}\right) \\
\quad+\frac{\partial \chi_{1}}{\partial x} \frac{\partial}{\partial x} \nabla^{2} \phi_{0}+\frac{\partial \chi_{1}}{\partial y} \frac{\partial}{\partial y} \nabla^{2} \phi_{0} \\
\quad+\lambda \nabla \cdot\left(\omega_{0} \frac{\partial}{\partial p} \nabla \cdot \phi_{0}\right)+\nabla^{2} \phi_{0} \cdot \nabla^{2} \chi_{1}-\lambda f \frac{\partial \omega_{1}}{\partial p}=0 \\
\frac{\partial}{\partial t}\left(\frac{\partial \phi_{1}}{\partial p}\right)-J\left(\frac{\partial \phi_{1}}{\partial p}, \phi_{0}\right)-J\left(\frac{\partial \phi_{0}}{\partial p}, \phi_{1}\right) \\
\quad+\frac{\partial \chi_{1}}{\partial x} \frac{\partial}{\partial x}\left(\frac{\partial \phi_{0}}{\partial p}\right)+\frac{\partial \chi_{1}}{\partial y} \frac{\partial}{\partial y}\left(\frac{\partial \phi_{0}}{\partial p}\right)+s \omega_{1}=0 \\
\nabla^{2} \chi_{2}+\lambda \frac{\partial \omega_{1}}{\partial p}=0
\end{array}\right.
$$

The second approximation of $\omega$-equation can be derived as follows from (43) and (45):

$$
\begin{aligned}
s V^{2} \omega_{1} & +\lambda f^{2} \frac{\partial^{2} \omega_{1}}{\partial p^{2}} \\
= & \nabla^{2}\left\{J\left(\frac{\partial \phi_{1}}{\partial p}, \phi_{0}\right)+J\left(\frac{\partial \phi_{0}}{\partial p}, \phi_{1}\right)\right. \\
& \left.-\frac{\partial \chi_{1}}{\partial x} \frac{\partial}{\partial x}\left(\frac{\partial \phi_{0}}{\partial p}\right)-\frac{\partial \chi_{1}}{\partial y} \frac{\partial}{\partial y}\left(\frac{\partial \phi_{0}}{\partial p}\right)\right\} \\
& -\frac{\partial}{\partial p}\left\{J\left(\nabla^{2} \phi_{1}, \phi_{0}\right)+J\left(\nabla^{2} \phi_{0}, \phi_{1}\right)\right. \\
& -\frac{\partial \chi_{1}}{\partial x} \frac{\partial}{\partial x} \nabla^{2} \phi_{0}-\frac{\partial \chi_{1}}{\partial y} \frac{\partial}{\partial y} \nabla^{2} \phi_{0} \\
& -\lambda \nabla \cdot\left(\omega_{0} \frac{\partial}{\partial p} \nabla \phi_{0}\right)-\nabla^{2} \phi_{0} \cdot \nabla^{2} \chi_{1} \\
& \left.+2 \frac{\partial}{\partial t}\left(\frac{\partial^{2} \phi_{0}}{\partial x^{2}} \frac{\partial^{2} \phi_{0}}{\partial y^{2}}-\left(\frac{\partial^{2} \dot{\varphi}_{0}}{\partial x \partial y}\right)^{2}\right)\right\}
\end{aligned}
$$

And the second approximation of the divergence equation becomes as

$$
\begin{aligned}
& -\frac{\partial}{\partial t} \nabla^{2} \chi_{1}+J\left(\nabla^{2} \chi_{1}, \phi_{0}\right) \\
& \quad+2\left(\frac{\partial^{2} \psi_{0}}{\partial x^{2}} \frac{\partial^{2} \psi_{1}}{\partial y^{2}}-2 \frac{\partial^{2} \psi_{0}}{\partial x \partial y} \frac{\partial^{2} \psi_{1}}{\partial x \partial y}+\frac{\partial^{2} \psi_{1}}{\partial x^{2}} \frac{\partial^{2} \psi_{0}}{\partial y^{2}}\right) \\
& +f \nabla^{2} \psi_{2}=\nabla^{2} \phi_{2}
\end{aligned}
$$

The first and second terms of (47) cannot be derived from the so-called balance equation and are relating to the individual change of the divergence. The approximate equations of higher order can in the same way be obtained. It will be inferred in the same way with the previous paragraph that the solutions of type (41) represent the meteorologically important ones and do not include the gravity-inertia waves.

\section{Energy conservation in the baroclinic case}

It can in the wellknown way be seen that the total energy continues to be conserved for the closed quasi-geostrophic model (42). Namely the following relation can be derived for the kinetic energy $K$ and the quantity $p_{A}$ which is corresponding to the available potential energy:

$$
\frac{\partial}{\partial t} \iiint\left(K+P_{A}\right) d \sigma=0
$$

where $d \sigma$ is a differential volume element and

$$
\begin{aligned}
K & =\frac{1}{2}\left(u^{2}+v^{2}\right) \\
P_{A} & =\frac{1}{2} \frac{\lambda}{s}\left(\frac{\partial \phi}{\partial p}\right)^{2}
\end{aligned}
$$

But the total energy conservation cannot exactly be derived from the primitive equations (39) when $s$ is assumed to be a function of $p$ only, but the following approximate relation with respect to the Rossby number can be obtained :

$$
\frac{\partial}{\partial t} \iiint\left(K+P_{A}\right) d \sigma=0(R)
$$

Now the energy conservation for the second approximate equations (45) will be discussed. If (41) is substituted into the (39) and the terms of order $R^{0}, R^{1}$ and $R^{2}$ are taken, the following equations will be obtained:

$$
\left\{\begin{array}{c}
\frac{\partial}{\partial t}\left(u_{0}+R u_{1}\right)+u_{0} \frac{\partial}{\partial x}\left(u_{0}+R u_{1}\right) \\
\quad+v_{0} \frac{\partial}{\partial y}\left(u_{0}+R u_{1}\right)-f v_{1}=-\frac{\partial \phi_{1}}{\partial x}+0(R) \\
\frac{\partial}{\partial t}\left(v_{0}+R v_{1}\right)+u_{0} \frac{\partial}{\partial x}\left(v_{0}+R v_{1}\right) \\
\quad+v_{0} \frac{\partial}{\partial y}\left(v_{0}+R v_{1}\right)+f u_{1}=-\frac{\partial \phi_{1}}{\partial y}+0(R) \\
\frac{\partial}{\partial t}\left(\frac{\partial \phi_{0}}{\partial p}+R \frac{\partial \phi_{1}}{\partial p}\right)+u_{0} \frac{\partial}{\partial x}\left(\frac{\partial \phi_{0}}{\partial p}+R \frac{\partial \phi_{1}}{\partial p}\right) \\
\quad+v_{0} \frac{\partial}{\partial y}\left(\frac{\partial \phi_{0}}{\partial p}+R \frac{\partial \phi_{1}}{\partial p}\right)+s \omega_{0}=0(R)
\end{array}\right.
$$

Journ. Met. Soc. Japan 


$$
\mid \frac{\partial u_{1}}{\partial x}+\frac{\partial v_{1}}{\partial y}+\lambda \frac{\partial \omega_{0}}{\partial p}=0
$$

From the first and second equations of (51), the following relation can be obtained, for the closed boundary conditions :

$$
\begin{aligned}
& \partial \partial \iiint \frac{1}{2}\left\{\left(u_{0}+R u_{1}\right)^{2}+\left(v_{0}+R v_{1}\right)^{2}\right\} d \sigma \\
& \quad \equiv \iiint f\left(v_{1} u_{0}-u_{1} v_{0}\right) d \sigma+0(R) \\
& \quad=\iiint f \phi_{0} \cdot \nabla^{2} \chi_{1} d \sigma+0(R)
\end{aligned}
$$

In the same way, from the third equation of (51), the following relation can be derived:

$$
\begin{aligned}
& \frac{\partial}{\partial t} \iiint \frac{1}{2 s}\left(\frac{\partial \phi_{0}}{\partial p}+R \frac{\partial \phi_{1}}{\partial p}\right)^{2} d \sigma \\
& \quad=\iiint \phi_{0} \frac{\partial \omega_{0}}{\partial p} d \sigma+0(R) \\
& \quad=-\iiint \frac{f}{\lambda} \phi_{0} \cdot \nabla^{2} \chi_{1} d \sigma+0(R)
\end{aligned}
$$

Therefore, from (52) and (53) the following energy equation may be obtained :

$$
\begin{gathered}
\frac{\partial}{\partial t} \iiint \frac{1}{2}\left\{\left(u_{0}+R u_{1}\right)^{2}+\left(v_{0}+R v_{1}\right)^{2}\right. \\
\left.\quad+\frac{\lambda}{s}\left(\frac{\partial \phi_{0}}{\partial p}+R \frac{\partial \phi_{1}}{\partial p}\right)^{2}\right\} d \sigma=0(R)
\end{gathered}
$$

This is the energy conservation equation for the second approximate solutions with respect to the Rossby number and the same as the one for the primitive equations (39). The energy equation for the higher approximate solutions will have the same feature with (50) or (54).

\section{Solution of the balance equation}

The balance equation was derived as a generalization of the geostrophic approximation by Charney (1955) and Bolin (1955). (23) and (43) in this paper are the approximate relations of the balance equation which were formed in deriving the quasi-geostrophic equations by the perturbation method. The mothod solving the balance equation has been studied by many authors (for instance, Miyakoda (1956, 1960)). In this paragraph the solution of the balance equation by the perturbation method will be inquired. When the solution was expressed in the power series of the Rossby number, whether the power series converges or not, will be discussed in the case of circular isobaric pattern. And when the series does not converge or the convergence is too slow, the method calculating the solution will be discussed. This method is the one explained in (10).

The balance equation may be written as follows, omitting the term of $\partial f^{\prime} / \partial y^{\prime}$ :

$$
f^{\prime} \nabla^{2} \psi^{\prime}+2\left(\frac{\partial^{2} \psi^{\prime}}{\partial x^{\prime 2}} \frac{\partial^{2} \psi^{\prime}}{\partial y^{\prime 2}}-\left(\frac{\partial^{2} \psi^{\prime}}{\partial x^{\prime} \partial y^{\prime}}\right)^{2}\right)=\nabla^{2} \phi^{\prime}
$$

This may be written in the non-dimensional form as follows:

$$
f \nabla^{2} \phi+2 R\left(\frac{\partial^{2} \psi}{\partial x^{2}} \frac{\partial^{2} \psi}{\partial y^{2}}-\left(\frac{\partial^{2} \psi}{\partial x \partial y}\right)^{2},=\nabla^{2} \phi\right.
$$

where $R$ is the Rossby number. The solution of this equation is assumed to be expanded in the power series of $R$ as follows:

$$
\phi=\phi_{0}+R \phi_{1}+R^{2} \phi_{2}+\cdots \cdots
$$

If this series is substituted into the equation (55) and the coefficients of the same order of $R$ are set equal, the following equations may be obtained:

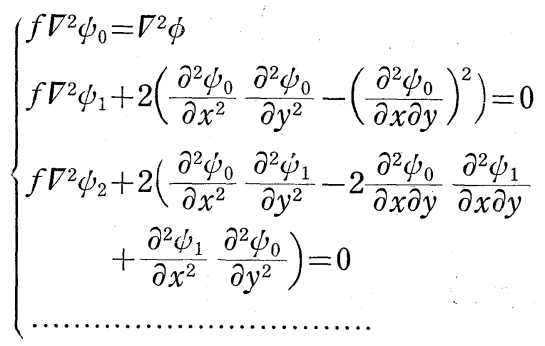

$\phi_{0}, \phi_{1}, \dot{\psi}_{2} \cdots \cdots$ can successively be solved from (57) for a given $\phi$. In order to examine the convergency of the series (56), the case of circular pattern will be discussed. Namely $\phi$ and $\phi$ are assumed to be functions of $r=\sqrt{x^{2}+y^{2}}$ only. Then the balance equation (55) may be written as follows:

$$
\frac{f}{r} \frac{\partial}{\partial r}\left(r \frac{\partial \psi}{\partial r}\right)+R \frac{1}{r} \frac{\partial}{\partial r}\left(\frac{\partial \psi}{\partial r}\right)^{2}=\frac{1}{r} \frac{\partial}{\partial r}\left(r \frac{\partial \phi}{\partial r}\right)
$$

In this case $\partial \dot{\psi}_{i} / \partial r(i=0,1,2, \cdots \cdots)$ can easily be solved as follows:

$$
\begin{aligned}
& \left.\frac{\partial \phi_{0}}{\partial r}=\frac{1}{f} \frac{\partial \phi}{\partial r}, \quad \frac{\partial \phi_{1}}{\partial r}=-\frac{1}{f^{3} r} \frac{\partial \phi}{\partial r}\right)^{2}, \\
& \frac{\partial \phi_{2}}{\partial r}=\frac{1}{f^{5} r^{2}}\left(\frac{\partial \phi}{\partial r}\right)^{3}, \quad \ldots \ldots \ldots \ldots \ldots \ldots
\end{aligned}
$$

Therefore, from (56),

$$
\begin{aligned}
v= & \frac{\partial \phi}{\partial r}=\frac{1}{f} \frac{\partial \phi}{\partial r}-R \frac{1}{f^{3} r}\left(\frac{\partial \phi}{\partial r}\right)^{2} \\
& +R^{2} \frac{2}{f^{5} r^{2}}\left(\frac{\partial \phi}{\partial r}\right)^{3}-R^{3} \frac{5}{f^{7} r^{3}}\left(\frac{\partial \phi}{\partial r}\right)^{4}+
\end{aligned}
$$




$$
=\frac{f r}{2 R}\left(-1+\sqrt{1+\frac{4 R}{f^{2} r} \frac{\partial \phi}{\partial r}}\right)
$$

This is the so-called gradient wind. The power series (59) converges when $\left|\frac{4 R}{f^{2} r} \frac{\partial \phi}{\partial r}\right|<1$, but this convergence is very. slow when $\left|\frac{4 R}{f^{2} r} \frac{\partial \phi}{\partial r}\right|$ is near to unity. When $\left|\frac{4 R}{f^{2} r} \frac{\partial \phi}{\partial r}\right|>1$, the gradient wind cannot be calculated by the series (59). In that case the gradient wind will be able to be calculated by using each term of the series (59) as follows. $R$ is assumed to be equal to $n \Delta R$ where $n$ is an integer and $4 R$ is sufficiently small. And the following calculating method is adopted :

$$
\begin{aligned}
(v)_{R=\Delta R}= & (v)_{R=0}+\Delta R\left(\frac{\partial v}{\partial R}\right)_{R=0} \\
(v)_{R=2 \Delta R}= & (v)_{R=\Delta R}+\Delta R\left(\frac{\partial v}{\partial R}\right)_{R=\Delta R} \\
= & (v)_{R=\Delta R}+\Delta R\left\{\left(\frac{\partial v}{\partial R}\right)_{R=0}\right. \\
& +\Delta R\left(\frac{\partial^{2} v}{\partial R^{2}}\right)_{R=0} \int^{i} \\
= & (v)_{R=0}+2 \Delta R\left(\frac{\partial v}{\partial R}\right)_{R=0} \\
& +(\Delta R)^{2}\left(\frac{\partial^{2} v}{\partial R^{2}}\right)_{R=0} \\
(v)_{R=3 \Delta R}= & (v)_{R=2 \Delta R}+\Delta R\left(\frac{\partial v}{\partial R}\right)_{R=2 \Delta R} \\
= & (v)_{R=0}+3 \Delta R\left(\frac{\partial v}{\partial R}\right)_{R=0} \\
& +3(\Delta R)^{2}\left(\frac{\partial^{2} v}{\partial R^{2}}\right)_{R=0} \\
& +(\Delta R)^{3}\left(\frac{\partial^{3} v}{\partial R^{3}}\right)_{R=0}
\end{aligned}
$$

and so on. By using (59), the above formula may be written as follows:

$$
\begin{aligned}
(v)_{R=\Delta R}= & \frac{1}{f} \frac{\partial \dot{\phi}}{\partial r}-(\Delta R) \frac{1}{f^{3} r}\left(\frac{\partial \phi}{\partial r}\right)^{2} \\
(v)_{R=2 \Delta R}= & \frac{1}{f} \frac{\partial \phi}{\partial r}-2(\Delta R) \frac{1}{f^{3} r}\left(\frac{\partial \phi}{\partial r}\right)^{2} \\
& +4(\Delta R)^{2} \frac{1}{f^{5} r^{2}}\left(\frac{\partial \phi}{\partial r}\right)^{3} \\
(v)_{R=3 \Delta R}= & \frac{1}{f} \frac{\partial \phi}{\partial r}-3(\Delta R) \frac{1}{f^{3} r}\left(\frac{\partial \phi}{\partial r}\right)^{2} \\
& +12(\Delta R)^{2} \frac{1}{f^{5} r^{2}}\left(\frac{\partial \phi}{\partial r}\right)^{3} \\
& -30(\Delta R)^{3} \frac{1}{f^{7} r^{3}}\left(\frac{\partial \phi}{\partial r}\right)^{4}
\end{aligned}
$$

and so on. This calculating formula will be compared with the power series (59) in the following. If $f=1, R=0.1$ and $r=0.8$ are taken, the series (59) does not converge when $|\partial \phi / \partial r|>1$. Even though it does not converge, the first three terms of it will here be used. Geostrophic wind $\partial \phi / \partial r$ and gradient wind (60) will be denoted by $v_{g}$ and $v_{r}$ respectively. Wind values calculated by the first three terms of the power series (59) and by the formula (65) will be denoted by $v_{1}$ and $v_{2}$ respectively. Then the following examples can be obtained (non-dimension).

\begin{tabular}{l|lllll}
\hline$v_{g}$ & 1.00 & 1.50 & 2.00 & 2.50 & 3.00 \\
$v_{r}$ & 0.83 & 1.16 & 1.46 & 1.74 & 2.00 \\
$v_{1}$ & 0.88 & 1.36 & 2.00 & 2.89 & 4.13 \\
$v_{2}$ & 0.81 & 1.15 & 1.50 & 1.91 & 2.44 \\
\hline
\end{tabular}

As may be seen from these values, $v_{2}$ is better than $v_{1}$ as an approximation of the gradient wind.

In general the same consideration with (61), (62) and (63) instead of the power series should be adopted in the perturbation method.

\section{Secular term and numerical calculation scheme}

As was mentioned in paragraph 3, when the secular term appears in the perturbation method, numerical calculation by the difference method should carefully be done. A simple example will here be discussed. The following ordinary differential equation will be considered :

$$
\frac{d^{2} \phi}{d t^{2}}+\alpha^{2} \phi=\varepsilon \phi
$$

where $\alpha$ and $\varepsilon$ are constants and $\phi$ is a function of $t$. The solution of (67) is assumed to be expanded in the power series of $\varepsilon$ as follows :

$$
\phi=\phi_{0}+\varepsilon \phi_{1}+\varepsilon^{2} \phi_{2}+\cdots \cdots
$$

Then the following equations for $\phi_{0}$ and $\phi_{1}$ may be obtained:

$$
\begin{aligned}
& \frac{d^{2} \phi_{0}}{d t^{2}}+\alpha^{2} \phi_{0}=0 \\
& \frac{d^{2} \phi_{1}}{d t^{2}}+\alpha^{2} \phi_{1}=\phi_{0}
\end{aligned}
$$

The approximate solution of (67) is assumed to be able to be expressed in the form $\phi=\phi_{0}$ $+\varepsilon \phi_{1}$. The solution of (69) with the initial 
conditions $\left(\phi_{0}\right)_{t=0}=1$ and $\left(d \phi_{0} / d t\right)_{t=0}=0$ may be obtained as follows :

$$
\phi_{0}=\cos \alpha t
$$

And the solution of (70) with the initial conditions $\left(\phi_{1}\right)_{t=0}=\left(d \phi_{1} / d t\right)_{t=0}=0$ becomes as follows :

$$
\phi_{1}=\frac{t}{2 \alpha} \sin \alpha t
$$

The solution of the equation with the initial conditions $(\phi)_{t=0}=1$ and $(d \phi / d t)_{t=0}=0$ may be obtained as follows :

$$
\begin{aligned}
\phi & =\phi_{0}+\varepsilon \phi_{1} \\
& =\cos \alpha t+\varepsilon \frac{t}{2 \alpha} \sin \alpha t
\end{aligned}
$$

But the exact solution of (67) is

$$
\phi=\cos \sqrt{\alpha^{2}-\varepsilon} t
$$

For the fixed small constant $\varepsilon$, (73) is equal to (74) only when $t$ is sufficiently small. Namely (69) and (70) may be regarded as a system of simultaneous equations only in the sufficiently small range of $t$. In extending range of $t$ by the difference method, in every time step a system of the simultaneous equations (69) and (70) should be solved as a new initial value problem. This method is always useful in the numerical integration by the perturbation method.

\section{Conclusion}

In this paper the solutions of the primitive equations was discussed by the perturbation method with respect to the Rossby number. It was first demonstrated in both the baro. tropic and baroclinic cases that the first approximate equations were the quasi-geostrophic equations and the approximate equations of higher order did not include the gravity-inertia waves. Next the total energy conservation was inquired and it was shown that though it was conserved in the quasigeostrophic system, but in the approximate system of higher order it was not conserved without omitting the quantity of order of the Rossby number. Finally the convergency of the perturbation method was examined especially for the balance equation and a calculation method was presented when it did not converge.

There are some important problems which were not discussed in this paper. One of them is the computational errors. The order of magnitudes of the higher order terms in the perturbation method is equal to that of the errors in the usual calculation scheme of the quasi-geostrophic equations. Therefore in order to obtain the solutions of higher order, a more precise calculation scheme will be necessary. And new criterion to check the computational instability will become necessary. The other problem is the term of static stability in the equation of thermodynamics. In this paper it was assumed to be a function of $p$ only. Then the total energy is not exactly conserved. These problems will in future be studied.

\section{Acknowledgement}

The author wishes to express his hearty thanks to Dr. H. Arakawa and Dr. S. Syōno for their kind guidances. He also wishes to express his hearty thanks to the staff members of his laboratory for their kind discussions.

\section{References}

Bolin, B., 1955: Numerical forecasting with the barotropic model. Tellus, 7, 27-49.

Charney, J., 1948: On the scale of atmospheric motions. Geofys. Publ., 17, Nr. 2.

Charney, J., 1955: The use of the primitive equations of motion in numerical prediction. Tellus, 7, 22-26.

Charney, J., 1960: (to be published)

Gambo, K., 1960: Geostrophic approximation related to the scale effect of atmospheric disturbances. Geophys. Mag., 29, 535-556.

Hinkelmann, K., 1959: Ein numerisches Experiment mit der primitiven Gleichungen. In the Atmosphere and Sea in Motion, 486-500, Rock. efeller Inst. Press, New York.

Kibel, I.A., 1958: A method of short-range prognosis of meteorological elements. (In Russian) Doklady Akad. Nauk SSSR, 118, 687-690.

Magata, M., 1960: On the approximate solutions of the barotropic vorticity equation. Journ. Met. Soc. Japan, 38, 80-93.

Miyakoda, K., 1956: On a method of solving the balance equation. Journ. Met. Soc. Japan, 34, 364-367.

Obukhov, A. M., 1949: On the problem of the geostrophic wind. (In Russian) Izv. Acad., Nauk SSSR, Ser. geograf. i geol., 4, 281-306.

Phillips, N.A., 1960: On the problem of initial data for the primitive equations. Tellus, 12, 121-126. 
Smagorinsky, .J., 1958: On the numerical integration of the primitive equation of motion in a closed region. Mon. Weath. Rev., 86, 457-466.
Yudin, M.I., 1959: Ways of developing a physical basis for a numerical short-range forecast. (In Russian) Meteor. i Gidrol., 2, 46-51.

\title{
摂動法による運動方程式の解について
}

\author{
曲田光 夫
}

(気象研究所)

運動方程式の各項のオーダーを次元解析によつて評価し, 解を Rossby 数のべき級数の形飞表現すると， $R^{1}$ の項 までの方程式が，いわゆる準地衡風近似の方程式であることが示された。またこの $R$ のべき級数の形の解が，慣性 重力波のような small scale の波を含まない地衡風的な解であり, small scale の解は $R$ のべき級数の形《注表現 できないことが，線型モデルの場合飞証明された。次飞解を表わす $R$ のべき級数が収束しない場合の扱い力が，特に balance equation の解飞ついて示された。このよろな方向で，準地衡風近似解より精度のよい解を得る手順が論じ られた。最後に，このような近似解が，安定度の係数の気圧のみの函数と仮定する限りでは， $R^{1}$ のオ一ダ一の項を 除いて，エネルギー保存の法則を満たすことが示された。 